\title{
Identifying Residual Nodal Disease in Sentinel Lymph Node Surgery After Neoadjuvant Chemotherapy for Breast Cancer
}

\author{
Judy C. Boughey, MD ${ }^{1}$ \\ Department of Surgery, Mayo Clinic, Rochester, MN
}

The use of sentinel lymph node surgery after neoadjuvant chemotherapy for patients who present with $\mathrm{cN} 1$ breast cancer provides an opportunity to avoid axillary lymph node dissection for those patients who have eradication of their nodal disease with chemotherapy. Since the initial publication of prospective trials demonstrating the false-negative rate of sentinel lymph node (SLN) surgery in this setting, this practice has been increasing. ${ }^{1-4} \mathrm{~A}$ recent survey of the American Society of Breast Surgeons (ASBrS) reported that $85 \%$ of respondents offered SLN surgery to some patients in this setting. ${ }^{5}$

\section{SLN MAPPING}

Laws et al. ${ }^{6}$ reported their experience at Dana-Farber/ Brigham and Women's Cancer Center (DF-BWCC) with 96 patients who underwent SLN for $\mathrm{cN} 1$ disease converted to $\mathrm{ycN} 0$ disease. The authors reported that patients who had failed SLN mapping had higher rates of nodal macrometastases $(69 \%$ for the 16 patients with 0 SLN identified) compared with those with one or more SLNs retrieved. This differs from the results of the ACOSOG Z1071 trial, which found no significant difference in the nodal positivity rate between those patients with failure to identify a SLN (26/50 patients-52\%) and those with SLN identification $(382 / 637$ patients-60\%, $p=0.30){ }^{7}$ Either way, failed SLN mapping is a clear indication for ALND in the setting of $\mathrm{cN}+$ disease before chemotherapy. SLN identification rates in this setting are highest with the use of

(C) Society of Surgical Oncology 2019

First Received: 18 June 2019;

Published Online: 19 August 2019

J. C. Boughey, MD

e-mail: boughey.judy@mayo.edu dual tracers to map the SLNs. ${ }^{7}$ Since dual tracer has highest SLN identification rate and decreases the false-negative rate, use of dual tracer is recommended when performing SLN surgery after neoadjuvant chemotherapy in a patient who presented with $\mathrm{cN} 1$ disease at diagnosis.

When performing SLN surgery, if the first SLN resected is found to be positive before resecting additional SLNs, the surgeon will usually continue directly to axillary dissection without removing additional SLNs or ensuring removal of a certain minimum number of SLNs. Therefore, the number of SLNs resected in cases with a positive SLN may not be an accurate reflection of the number of SLNs present, and what is important is that a positive node was identified. While the DF-BWCC group defined adequate mapping as resecting 3 or more SLNs, of the 16 patients with 1 or 2 SLNs resected, which were defined as failed mapping, 8 patients had a positive SLN—reflecting that the SLN technique worked well. Therefore, adequate staging information was obtained in 72 patients (64 plus 8) or $75 \%$ of the patient cohort.

While some centers prefer the resection of a minimum of 3 SLNs, many are comfortable with two SLNs, acknowledging that while the greater the number of SLNs resected the lower the FNR, it is not feasible to identify three SLNs in all patients. Although the initial data from the clinical trials recommended resecting two SLNs (and that greater than 2 SLNs was better), with further advancements and use of complementary modalities (such as placing a clip, use of IHC etc.) to decrease the FNR, an acceptable FNR can be achieved with these approaches and is not dependent solely on the number of SLNs analyzed. $^{1-3}$

In the DF-BWCC series, clips were not placed in patients with positive nodes at diagnosis; however, most centers have adopted this practice; $67 \%$ of the ASBrS surgeons surveyed reported that they routinely have 
a clip placed in patients with biopsy-proven positive nodes. ${ }^{5}$ With adoption of marking the positive node (with a clip) and resecting the "clipped" node, the FNR is much lower and the number of SLNs resected is less important. In fact in the Netherlands, the MARI procedure (Marking the Axilla with Radioactive Rodine seed) only removes the marked biopsy-proven positive node; therefore, a single lymph node is resected and the FNR is $7 \% .^{8}$ Removal of just one lymph node that contains the marker may be adequate.

The overall reported rate in this study of sparing ALND in 24 of 96 patients $(25 \%)$ was lower that reported in other series. The MSKCC group reported avoiding ALND in 62 of 128 (48\%) patients, which was similar to Mayo Clinic Rochester where ALND was avoided in $48 \%$ of 193 patients treated with SLN surgery after NAC with low regional recurrence rates with short follow-up. ${ }^{4,9}$ The lower proportion of patients spared ALND may be related to the requirement of resecting three SLNs. The proportion of patients with negative axillary nodes also varies by response rates, which are dependent on tumor biology and type and length of systemic therapy delivered and will change over time with advances in systemic therapy. It remains important that surgeons adopting SLN in these patients are performing a thorough evaluation of the axilla, including palpation and resection of palpably abnormal nodes, and for cases with failed mapping, ALND should be performed.

\section{INTRAOPERATIVE SLN ASSESSMENT}

Intraoperative assessment of sentinel lymph nodes provides an opportunity to identify positive nodes at the time of surgery and proceed directly to an axillary lymph node dissection, therefore decreasing the need for a subsequent operation. Intraoperative pathological assessment is associated with possible false negatives in all settings. In the setting of sentinel lymph node surgery without any neoadjuvant therapy, intraoperative assessment is more reliable in cases with larger nodal metastasis and in cases with invasive ductal carcinoma. It is known to be more challenging to identify small foci of nodal disease, such as micrometastasis and isolated tumor cells as well as nodal metastasis in invasive lobular carcinoma with rapid intraoperative assessment. ${ }^{10,11}$ It is therefore not surprising, as nicely shown in the paper by Laws et al., that smaller foci of nodal disease in the postneoadjuvant setting are associated with a higher intraoperative false-negative rate. Use of immunohistochemical (IHC) staining of the sentinel nodes decreases the false-negative rate and detects smaller foci of disease in the nodes, including isolated tumor cells. ${ }^{2}$ Nodal disease identified by IHC is more likely to be missed on intraoperative analysis.

All patients with breast cancer undergoing sentinel lymph node surgery are counselled regarding the possible results_-negative nodes versus positive node(s) - and the impact that the result would have on further treatment recommendations: further axillary surgery, adjuvant radiation, etc. The timing of further axillary surgeryimmediate or a subsequent operation-also is something that should be routinely discussed at centers using intraoperative assessment of the sentinel lymph nodes, along with the potential for negative intraoperative findings and positive nodes on final pathology. This is similar to the routine discussion regarding margins of a breast cancer lumpectomy. Surgeons should be aware of the higher potential for negative intraoperative findings with low volume axillary nodal disease, especially in the postneoadjuvant chemotherapy setting and ensure that patients are aware of the possibility of needing a second surgery.

In addition to false negatives, there is the possibility of false-positive intraoperative assessment of sentinel lymph nodes. False-positive intraoperative sentinel nodes have been reported in the setting of a hydroMARK clip placed in the lymph node at initial diagnosis. This was thought to be due to mild atypia and mitosis seen on intraoperative analysis, which was in fact a reaction to the hydroMARK gel causing cavity formation with mucoid material and epithelioid proliferation. ${ }^{12}$

\section{PATIENT SELECTION FOR SLN SURGERY}

The authors demonstrate that axillary ultrasound after neoadjuvant chemotherapy is not helpful in assessing axillary response after NAC and recommend physical examination of the axilla to select patients for SLN surgery after NAC. While the data demonstrate that axillary ultrasound evaluating for normal or abnormal lymph nodes does not aid in the identification of patients with negative axillary nodes, it remains a tool that can be used along with other information to guide decision making. ${ }^{12}$ While normal axillary nodes on ultrasound are not required to consider SLN surgery, the finding of multiple highly suspicious lymph nodes can be helpful to educate the patient regarding the higher likelihood of finding positive nodes, particularly if in the setting of a significant volume of residual disease in the breast and tumor biology associated with lower rates of complete response. This also can allow the surgeon to consider approaching the SLN surgery first to send the SLNs to pathology earlier during the procedure, or consider ALND. 
While studies have shown that technique of sentinel lymph node surgery is critical for the accuracy of the procedure in this setting, patient selection also is important. Patients with HER2-positive disease and those with triplenegative breast cancer have higher rates of pathologic complete response to neoadjuvant chemotherapy. They also have the highest rates of conversion from biopsyproven, node-positive disease before chemotherapy to pathologically node-negative disease after neoadjuvant chemotherapy (64.7\% in HER2-positive breast cancer and 49.4\% in triple-negative breast cancer in ACOSOG Z1071 trial). ${ }^{13}$ Additionally, patients with eradication of disease in the breast have higher rates of eradication of disease in the lymph nodes. ${ }^{14}$

Use of sentinel lymph node surgery in patients with node-positive breast cancer treated with neoadjuvant chemotherapy is not recommended for all patients. It is not appropriate for a "one size fits all" approach. Patients who have a suboptimal response to neoadjuvant chemotherapy, with disease progression, stable disease, or minimal decrease in the primary tumor size are not the best candidates for consideration of sentinel lymph node surgery, because these patients are at high risk for having persistent nodal disease. In this setting, axillary lymph node dissection remains a good option allowing the resection of the axillary lymph nodes for staging and local control. The benefit of sentinel lymph node surgery is to allow those patients who have converted to pathologically node-negative disease to avoid axillary lymph node dissection. Therefore, the best patients for selection for this procedure are those patients who have a good clinical response to neoadjuvant chemotherapy and are most likely to be pathologically node-negative. Appropriate patient selection can help to minimize the potential for false negatives.

\section{RECOMMENDATIONS}

Patients with node-positive breast cancer being recommended treatment with neoadjuvant chemotherapy should have a clip placed in the positive axillary lymph node as well as the breast tumor at time of initial diagnosis. After completion of all planned neoadjuvant therapy, they should undergo physical examination and imaging to assess response of the tumor in the breast and in the lymph nodes. When considering use of SLN surgery, the surgeon should take into consideration the extent of nodal disease at presentation, tumor biology, and likelihood of nodal response along with the patient's clinical and imaging response in both the breast and axilla. Discussion with the patient regarding SLN surgery should include plans for (1) failure to identify any SLNs, (2) identification of a positive SLN, and (3) in cases with a clip marking the positive node failure to identify the clipped node. If intraoperative assessment of the SLNs is available, the possibility of changes on final pathology should be discussed. Use of dual tracers is recommended, along with resection of all blue lymph nodes, all radioactive nodes $(>10 \%$ of greatest count) and any palpably abnormal lymph nodes. In cases where the positive node was marked (clipped or tattooed), efforts should be made to ensure resection of that node.

The I-SPY trial developed surgical standards for SLN surgery following neoadjuvant chemotherapy obtained by consensus across the I-SPY centers. ${ }^{15}$ For patients with node-positive disease at presentation, these recommend placement of a clip in the positive node at diagnosis. In cases where a clip was placed, resection of the clipped node is required, and there is no recommended minimum number of SLNs resected. If the clipped node is not resected, ALND is recommended. In cases without a clip placed, a minimum of two SLNs are required to be resected; if this is not achieved, ALND is required. In addition, all palpably abnormal lymph nodes should be resected for pathological evaluation.

\section{CONCLUSIONS}

SLN surgery after NAC is an opportunity to spare patients with a favorable response to NAC from an ALND. However, this is not a one size fits all paradigm and should not be applied uniformly to all node-positive patients treated with NAC. Patient selection based on response is important. Technique of SLN surgery is crucial to minimize the FNR and should be viewed as a toolbox; use of dual tracer, resection of "clipped" lymph node, comprehensive evaluation of the axilla to resect all SLNs, and use of immunohistochemistry on the SLNs can all help to decrease the FNR. Intraoperative assessment of the SLNs allows patients to proceed to ALND at one setting but is associated with potential false negatives, and patients should be aware of this possibility in this setting (and in all settings). Outside of clinical trials, ALND remains the standard for patients with any size of nodal metastases in a SLN after NAC, given the high rates of positive non-SLNs, until further data becomes available.

\section{REFERENCES}

1. Boughey JC, Suman VJ, Mittendorf EA, et al. Sentinel lymph node surgery after neoadjuvant chemotherapy in patients with node-positive breast cancer: the ACOSOG Z1071 (Alliance) clinical trial. JAMA. 2013;310(14):1455-61.

2. Boileau JF, Poirier B, Basik M, Holloway CM, Gaboury L, Sideris L, et al. Sentinel node biopsy following neoadjuvant chemotherapy in biopsy proven node positive breast cancer: the SN FNAC Study. J Clin Oncol. 2013;31(suppl):abstr 1018. 
3. Kuehn T, Bauerfeind I, Fehm T, et al. Sentinel-lymph-node biopsy in patients with breast cancer before and after neoadjuvant chemotherapy (SENTINA): a prospective, multicentre cohort study. Lancet Oncol. 2013;14(7):609-18.

4. Nguyen TT, Hoskin TL, Day CN, Degnim AC, Jakub JW, Hieken TJ, Boughey JC. Decreasing use of axillary dissection in nodepositive breast cancer patients treated with neoadjuvant chemotherapy. Ann Surg Oncol. 2018;25(9):2596-602.

5. Caudle AS, Bedrosian I, Milton DR,DeSnyder SM, Kuerer HM, Hunt KK, Mittendorf EA. Use of sentinel lymph node dissection after neoadjuvant chemotherapy in patients with node-positive breast cancer at diagnosis: practice patterns of American Society of Breast Surgeons Members. Ann Surg Oncol. 2017;24(10):2925-34.

6. Laws $\mathrm{AH}$, Hughes $\mathrm{ME}, \mathrm{Hu} \mathrm{J}$, et al. Impact of residual nodal disease burden on technical outcomes of sentinel lymph node biopsy in node-positive (cN1) breast cancer patients treated with neoadjuvant chemotherapy. Ann Surg Oncol. 2019 (in press).

7. Boughey JC, Suman VJ, Mittendorf EA, et al. Factors affecting sentinel lymph node identification rate after neoadjuvant chemotherapy for breast cancer patients enrolled in ACOSOG Z1071 (alliance). Ann Surg. 2015;261(3):547-52.

8. Donker M Straver ME, Wesseling J, et al. Marking axillary lymph nodes with radioactive iodine seeds for axillary staging after neoadjuvant systemic treatment in breast cancer patients: the MARI Procedure. Ann Surg. 2015;261(2):378-82.

9. Mamtani A, Barrio AV, King TA, et al. How often does neoadjuvant chemotherapy avoid axillary dissection in patients with histologically confirmed nodal metastases? Results of a prospective study. Ann Surg Oncol. 2016;23(11):3467-74.
10. Akay CL, Albarracin C, Torstenson T, et al. Factors impacting the accuracy of intra-operative evaluation of sentinel lymph nodes in breast cancer. Breast J. 2018;24(1):28-34.

11. Jensen AJ, Naik AM, Pommier RF, Vetto JT, Troxell ML. Factors influencing accuracy of axillary sentinel lymph node frozen section for breast cancer. Am J Surg. 2010;199(5):629-35.

12. Nguyen TT, Hieken TJ, Glazebrook KN, Boughey JC. Localizing the clipped node in patients with node-positive breast cancer treated with neoadjuvant chemotherapy: early learning experience and challenges. Ann Surg Oncol. 2017;24(10):3011-6.

13. Boughey JC, McCall LM, Ballman KV, et al. Tumor biology correlates with rates of breast-conserving surgery and pathologic complete response after neoadjuvant chemotherapy for breast cancer: findings from the ACOSOG Z1071 (Alliance) Prospective Multicenter Clinical Trial. Ann Surg. 2014;260(4):608-14; discussion 614-6.

14. Barron AU, Hoskin TL, Day CN, Hwang ES, Kuerer HM, Boughey JC. Association of low nodal positivity rate among patients with ERBB2-positive or triple-negative breast cancer and breast pathologic complete response to neoadjuvant chemotherapy. JAMA Surg. 2018;153(12):1120-6.

15. Boughey JC, Alvarado MD, Lancaster RB, et al. Surgical standards for management of the axilla in breast cancer clinical trials with pathological complete response endpoint. NPJ Breast Cancer. 2018;4:26.

Publisher's Note Springer Nature remains neutral with regard to jurisdictional claims in published maps and institutional affiliations. 